TRANSACTIONS OF THE

AMERICAN MATHEMATICAL SOCIETY

Volume 361, Number 3, March 2009, Pages 1173-1188

S 0002-9947(08)04653-9

Article electronically published on October 9, 2008

\title{
BEREZIN TRANSFORMS ON PLURIHARMONIC BERGMAN SPACES
}

\author{
MIROSLAV ENGLIŠ
}

\begin{abstract}
We show that, perhaps surprisingly, in several aspects the behaviour of the reproducing kernels of Toeplitz operators and of the Berezin transform on some weighted pluriharmonic Bergman spaces is the same as in the holomorphic case.
\end{abstract}

\section{INTRODUCTION}

Let $\Omega$ be a bounded domain in $\mathbf{C}^{n}, L_{\text {hol }}^{2}(\Omega) \subset L^{2}(\Omega)$ the Bergman space of all square-integrable holomorphic functions on $\Omega$, and $K(x, y)$ its reproducing kernel, i.e. the Bergman kernel. Thus

$$
f(x)=\int_{\Omega} f(y) K(x, y) d y=\left\langle f, K_{x}\right\rangle, \quad K_{x}:=K(\cdot, x),
$$

for all $f \in L_{\text {hol }}^{2}$ and $x \in \Omega$. Recall that for $\phi \in L^{\infty}(\Omega)$, the Toeplitz operator $T_{\phi}$ with symbol $\phi$ is defined by

$$
T_{\phi}: L_{\mathrm{hol}}^{2} \rightarrow L_{\mathrm{hol}}^{2}, \quad T_{\phi} f:=P(\phi f),
$$

where $P: L^{2} \rightarrow L_{\text {hol }}^{2}$ is the orthogonal projection (the Bergman projection). The Berezin symbol of a (bounded linear) operator $T$ on $L_{\text {hol }}^{2}$ is, by definition, the function $\widetilde{T}$ on $\Omega$ defined by

$$
\widetilde{T}(x):=\frac{\left\langle T K_{x}, K_{x}\right\rangle}{K(x, x)}=\left\langle T \frac{K_{x}}{\left\|K_{x}\right\|}, \frac{K_{x}}{\left\|K_{x}\right\|}\right\rangle .
$$

Finally, the Berezin transform of $f \in L^{\infty}$ is, by definition, the Berezin symbol of the Toeplitz operator $T_{f}$ :

$$
B f(x)=\widetilde{T_{f}}(x)=K(x, x)^{-1} \int_{\Omega} f(y)|K(x, y)|^{2} d y
$$

It is immediate that the mapping $T \mapsto \widetilde{T}$ is linear, $\widetilde{I}=\mathbf{1},\left(T^{*}\right)^{\sim}=\overline{\widetilde{T}},\|\widetilde{T}\|_{\infty} \leq\|T\|$, and $\widetilde{T}$ is a real-analytic function on $\Omega$; similarly for $f \mapsto B f$. Since the function $\left\langle T K_{y}, K_{x}\right\rangle$, being holomorphic in $x$ and $\bar{y}$, is uniquely determined by its restriction to the diagonal $x=y$, it also follows that both mappings $T \mapsto \widetilde{T}$ and $f \mapsto B f$ are one-to-one - a fact which is of crucial importance for some applications.

Received by the editors May 15, 2006.

2000 Mathematics Subject Classification. Primary 47B35; Secondary 32A36, 31C10, 41A60.

Key words and phrases. Berezin transform, pluriharmonic Bergman kernel.

This research was supported by GA AV ČR grant no. A1019304 and by AV ČR IRP no. AV0Z10190503.

(C)2008 American Mathematical Society Reverts to public domain 28 years from publication 
There are also the weighted analogues of all the above objects: namely, for any continuous, positive weight function $w$ on $\Omega$, the subspace $L_{\mathrm{hol}}^{2}(\Omega, w)$ of all holomorphic functions in $L^{2}(\Omega, w)$ is closed and possesses a reproducing kernel $K_{w}(x, y)$, the weighted Bergman kernel, and one may define the Toeplitz operators $T_{f}^{(w)}$, Berezin symbols $\widetilde{T}^{(w)}$ and Berezin transform $B^{(w)}$ in the same way as before.

Now consider a strictly plurisubharmonic (or strictly- $P S H$ for short) real-valued smooth function $\Phi$ on $\Omega$. Then $g_{i \bar{j}}=\partial^{2} \Phi / \partial z_{i} \partial \bar{z}_{j}$ defines a Kähler metric on $\Omega$, with the associated volume element $d \mu(z)=\operatorname{det}\left[g_{i \bar{j}}\right] d z$ ( $d z$ being the Lebesgue measure). For any $h>0$, we then have, in particular, the weighted Bergman spaces $L_{\mathrm{hol}}^{2}\left(\Omega, e^{-\Phi / h} d \mu\right)=: L_{\mathrm{hol}, h}^{2}$, the corresponding reproducing kernels $K_{h}(x, y)$, Toeplitz operators $T_{f}^{(h)}$, and the Berezin transforms $B_{h} f$. It turns out that the following theorem holds. (Recall that $\rho \in C^{\infty}(\bar{\Omega})$ is called a defining function for $\Omega$ if $\rho>0$ on $\Omega$, and $\rho=0,\|\nabla \rho\| \neq 0$ on $\partial \Omega$. The definition of and basic facts about bounded symmetric domains are reviewed in Section 3 below.)

Theorem Q ([E2, $\mathrm{BMS}], \mathrm{Ber}, \overline{\mathrm{Cob}}, \overline{\mathrm{BLU}})$. Assume that $\Omega \subset \mathbf{C}^{n}$ is smoothly bounded and strictly pseudoconvex and $e^{-\Phi}$ is a defining function for $\Omega$, or that $\Omega$ is a bounded symmetric domain in $\mathbf{C}^{n}$ and $e^{\Phi}$ is the (unweighted) Bergman kernel of $\Omega$, or that $\Omega=\mathbf{C}^{n}$ and $\Phi(z)=|z|^{2}$. Then as $h \searrow 0$, there are asymptotic expansions

$$
\begin{aligned}
& K_{h}(x, x) \approx e^{\Phi(x) / h} h^{-n} \sum_{j=0}^{\infty} h^{j} b_{j}(x) ; \\
& B_{h} f \approx \sum_{j=0}^{\infty} h^{j} Q_{j} f ; \quad \text { and } \\
& T_{f}^{(h)} T_{g}^{(h)} \approx \sum_{j=0}^{\infty} h^{j} T_{C_{j}(f, g)}^{(h)} \quad \text { (in operator norm) },
\end{aligned}
$$

for some functions $b_{j} \in C^{\infty}(\Omega)$, with $b_{0}=1$; some differential operators $Q_{j}$, with $Q_{0}=I$ and $Q_{1}$ the Laplace-Beltrami operator with respect to the metric $g_{i \bar{j}}$; and some bidifferential operators $C_{j}$, where $C_{0}(f, g)=f g$ and $C_{1}(f, g)-C_{1}(g, f)=$ $\frac{i}{2 \pi}\{f, g\}$ (the Poisson bracket of $f$ and $g$ with respect to the metric $g_{i \bar{j}}$ ).

The last theorem has an elegant application to quantization on Kähler manifolds. Recall that the traditional problem of quantization consists in looking for a map $f \mapsto Q_{f}$ from $C^{\infty}(\Omega)$ into operators on some (fixed) Hilbert space which is linear, conjugation-preserving, $Q_{\mathbf{1}}=I$, and as the Planck constant $h \searrow 0$,

$$
\left[Q_{f}, Q_{g}\right] \approx \frac{i h}{2 \pi} Q_{\{f, g\}}
$$

(The spectrum of $Q_{f}$ is then interpreted as the possible outcomes of measuring the observable $f$ in an experiment, and (4) amounts to a correct semiclassical limit.) Theorem Q implies that (4) holds for $Q_{f}=T_{f}^{(h)}$, the Toeplitz operators on the Bergman spaces $L_{\mathrm{hol}, h}^{2}$. This is the so-called Berezin-Toeplitz quantization.

There is also another approach to quantization, discarding the operators $Q_{f}$ but rather looking for a noncommutative associative product $*$ on $C^{\infty}(\Omega)$, depending 
on $h$, such that as $h \searrow 0$,

$$
f * g \rightarrow f g, \quad \frac{f * g-g * f}{h} \rightarrow \frac{i}{2 \pi}\{f, g\} .
$$

Such products are called star-products and are the subject of deformation quantization. The relationship to Bergman spaces is the following: in view of the injectivity of the map $T \mapsto \widetilde{T}$ from operators to their Berezin symbols, we can define for two bounded operators $T, U$ on $L_{\mathrm{hol}, h}^{2}$ a "product" of their symbols by

$$
\widetilde{T} * \widetilde{U}:=\widetilde{T U} .
$$

This gives a noncommutative associative product on

$$
\left\{\widetilde{T}: T \text { a bounded operator on } L_{\mathrm{hol}, h}^{2}\right\} \subset C^{\omega}(\Omega) .
$$

It can be shown from part (2) of Theorem Q (the asymptotics of $B_{h}$ ) that if $h$ is made to vary, these products can be glued into a star-product on $C^{\infty}(\Omega)$. This is the so-called Berezin quantization.

From the point of view of these applications, the weighted Bergman spaces $L_{\mathrm{hol}, h}^{2}$ have an obvious disadvantage in that their very definition requires a holomorphic structure (hence, in particular, they can make sense only on Kähler manifolds). On the other hand, the other ingredients - the operator symbols, the Toeplitz operators and the Berezin transform - make sense not only for $L_{\text {hol }}^{2}$, but for any subspace of $L^{2}$ with reproducing kernel. Hence it seems very natural to investigate whether any such spaces other than weighted Bergman spaces can be used for quantization.

For instance, one such candidate might be the harmonic Bergman spaces $L_{\text {harm }}^{2}$ of all harmonic functions in $L^{2}$. As in the holomorphic case, these possess a reproducing kernel, the harmonic Bergman kernel $H(x, y)$; in contrast to the usual Bergman kernel, $H(x, y)$ is real-valued and symmetric, $H(x, y)=H(y, x) \in \mathbf{R}$. Similarly, one has pluriharmonic Bergman spaces $L_{\mathrm{ph}}^{2}$ (and pluriharmonic Bergman kernels) consisting of all functions $f$ in $L^{2}$ for which $\partial^{2} f / \partial z_{j} \partial \bar{z}_{k}=0 \forall j, k$.

Unfortunately, it turns out that - from the point of view of the quantization applications at least - bad things happen. First of all, recall that for the BerezinToeplitz quantization we needed the Toeplitz operators to satisfy

$$
\frac{1}{h}\left[T_{f}^{(h)}, T_{g}^{(h)}\right] \approx \frac{i}{2 \pi} T_{\{f, g\}}^{(h)} \quad \text { as } h \searrow 0 .
$$

However, for Toeplitz operators on $L_{\text {harm }}^{2}(\Omega)$, this fails even on $\Omega=\mathbf{D}$, the unit disc in $\mathbf{C}$, with the hyperbolic metric (given by the Kähler potential $\Phi(z)=$ $\left.\log \frac{1}{1-|z|^{2}}\right)$ and $f(z)=z, g(z)=\bar{z}$. Second, recall that the Berezin quantization was based on the fact that the correspondence $T \mapsto \widetilde{T}$ between operators and their symbols was one-to-one. However, this fails on any harmonic Bergman space: if $f, g$ are any two linearly independent elements in $L_{\text {harm }}^{2}$, then the operator $T=\langle\cdot, \bar{f}\rangle g-\langle\cdot, \bar{g}\rangle f$ is easily seen to satisfy $\left\langle T H_{x}, H_{x}\right\rangle=f(x) g(x)-g(x) f(x)=0 \forall x ;$ hence $\widetilde{T} \equiv 0$, while apparently $T \neq 0$. Thus, there is no hope to perform the Berezin quantization either. See [E3] for the details.

In view of these failures, it would be only natural to expect that the other assertions (11)-(2) of Theorem Q (i.e. the asymptotics of the reproducing kernels and of the Berezin transform), or the injectivity of the map $f \mapsto B f$, would also 
very likely break down. The following results therefore came as some surprise for the author.

Recall that a domain $\Omega \subset \mathbf{C}^{n}$ is called complete circular if $x \in \Omega$ implies that $z x \in \Omega$ for all $z \in \mathbf{C},|z| \leq 1$. In particular, such domains are invariant under the rotations

$$
z \mapsto z e^{i \theta}, \quad \theta \in \mathbf{R} .
$$

Theorem 1. Let $\Omega \subset \mathbf{C}^{n}$ be complete circular and let $\nu$ be any finite measure on $\Omega$ invariant under the rotations (5). Then on $L_{\mathrm{ph}}^{2}(\Omega, d \nu)$,

$$
\widetilde{T}_{f}=0 \Longrightarrow T_{f}=0 \text {, }
$$

(i.e. $B f=0 \Longrightarrow f=0$ ).

Thus, although the Berezin symbol map $T \mapsto \widetilde{T}$ is not injective on all operators, it is injective on Toeplitz operators.

Theorem 2. Let $\Omega \subset \mathbf{C}^{n}$ and $\Phi$ be such that (1) holds, and assume in addition that $\Omega$ is complete circular and that $\Phi$ is invariant under the rotations (5) and tends to $+\infty$ at the boundary and (if $\Omega$ is unbounded) at infinity. Then the pluriharmonic Bergman kernels $H_{h}(x, y)$ of the spaces $L_{\mathrm{ph}}^{2}\left(\Omega, e^{-\Phi / h} d \mu\right)$ also have an asymptotic expansion of the form (11) as $h \searrow 0$ : namely,

$$
H_{h}(x, x) \approx e^{\Phi(x) / h} h^{-n} \sum_{j=0}^{\infty} h^{j} \beta_{j}(x),
$$

where the coefficients $\beta_{j}$ are related to those in (11) by

$$
\beta_{j}(x)= \begin{cases}2 b_{j}(x) & \text { if } x \neq 0, \\ b_{j}(x) & \text { if } x=0 .\end{cases}
$$

In other words, as long as everything is circularly symmetric, all goes fine with the asymptotics of the pluriharmonic Bergman kernels whenever it goes fine with the asymptotics of the corresponding holomorphic Bergman kernels. Finally, the same turns out to be true for the asymptotics of the Berezin transform.

Theorem 3. Consider the following spaces:

$$
\begin{aligned}
& L_{\text {harm }}^{2}\left(\mathbf{D},\left(1-|z|^{2}\right)^{1 / h}\right), \\
& L_{\mathrm{ph}}^{2}\left(\mathbf{C}^{n}, e^{-|z|^{2} / h}\right)
\end{aligned}
$$

(i.e. the harmonic Bergman spaces on the disc with respect to the usual weights and the pluriharmonic Fock spaces on $\mathbf{C}^{n}$ ), and also the pluriharmonic analogues of the standard weighted Bergman spaces on bounded symmetric domains in $\mathbf{C}^{n}$ in their Harish-Chandra realization. Then the associated Berezin transforms possess the asymptotic expansion (21), i.e. there exist differential operators $Q_{j}$ such that $\forall f \in C^{\infty} \cap L^{\infty}$,

$$
B_{h} f(x)=\sum_{j=0}^{\infty} h^{j} Q_{j} f(x) \quad \text { as } h \searrow 0 .
$$

In fact, these are the same $Q_{j}$ as in the holomorphic case. 
The proofs of these theorems go by explicit calculations of the reproducing kernels in question (which are possible owing to the rotational symmetry of the domains and measures) and by the method of stationary phase. The proofs of Theorems 12 can be found in Section 2, which also briefly reviews the relevant material on the method of stationary phase. Theorem 3 is proved in Section 3 , after briefly recalling the basic facts about bounded symmetric domains. The final section, Section 4 , contains some concluding remarks.

\section{Proofs of Theorems 1 12}

Throughout this section, let $\Omega$ be a bounded complete circular domain in $\mathbf{C}^{n}$ and $\nu$ a finite rotation-invariant measure on $\Omega$. Note first of all that $\Omega$ is star-like with respect to the origin, and therefore any pluriharmonic function on $\Omega$ can be uniquely written in the form

$$
f+\bar{g}, \quad f, g \text { holomorphic, } g(0)=0 .
$$

In view of the circularity of $\Omega$ and $\nu$, we have for any holomorphic function $F$ in $L^{1}(\Omega, d \nu)$,

$$
\int_{\Omega} F(z) d \nu(z)=\int_{0}^{2 \pi} \int_{\Omega} F\left(z e^{i \theta}\right) d \nu(z) \frac{d \theta}{2 \pi}
$$

However, if $F(z)=\sum_{\alpha \text { multiindex }} F_{\alpha} z^{\alpha}$ is the Taylor expansion of $F$, then

$$
\int_{0}^{2 \pi} F\left(z e^{i \theta}\right) \frac{d \theta}{2 \pi}=\int_{0}^{2 \pi} \sum_{\alpha} F_{\alpha} z^{\alpha} e^{i \theta|\alpha|} \frac{d \theta}{2 \pi}=F_{0}=F(0) .
$$

Thus

$$
\int_{\Omega} F(z) d \nu(z)=\nu(\Omega) \cdot F(0)
$$

It follows, first, that the reproducing kernel of $L_{\text {hol }}^{2}(\Omega, d \nu)$ at the origin is given by

$$
K_{0}(x) \equiv K(x, 0)=\frac{1}{\nu(\Omega)} \quad \forall x \in \Omega,
$$

and, second, that for any pluriharmonic function (7),

$$
\int_{\Omega}|f+\bar{g}|^{2} d \nu=\int_{\Omega}|f|^{2} d \nu+\int_{\Omega}|g|^{2} d \nu+2 \operatorname{Re} \nu(\Omega) f(0) g(0)=\|f\|^{2}+\|g\|^{2}
$$

since $g(0)=0$. Thus $f+\bar{g} \in L_{\mathrm{ph}}^{2}(\Omega, d \nu)$ if and only if $f, g \in L_{\text {hol }}^{2}(\Omega, d \nu)$. Now for any $x \in \Omega$, the function $H_{x}:=K_{x}+\overline{K_{x}-\frac{1}{\nu(\Omega)}} \in L_{\mathrm{ph}}^{2}(\Omega, d \nu)$ satisfies

$$
\begin{aligned}
\left\langle f+\bar{g}, H_{x}\right\rangle & =\int_{\Omega}(f+\bar{g})\left(\overline{K_{x}}+K_{x}-\frac{1}{\nu(\Omega)}\right) d \nu \\
& =\left\langle f, K_{x}\right\rangle+\int_{\Omega} f \cdot\left(K_{x}-\frac{1}{\nu(\Omega)}\right) d \nu+\overline{\int_{\Omega} g\left(K_{x}-\frac{1}{\nu(\Omega)}\right) d \nu}+\left\langle K_{x}, g\right\rangle \\
& =f(x)+\nu(\Omega) f(0)\left(K_{x}(0)-\frac{1}{\nu(\Omega)}\right)+\overline{\nu(\Omega) g(0)\left(K_{x}(0)-\frac{1}{\nu(\Omega)}\right)}+\overline{g(x)} \\
& =(f+\bar{g})(x),
\end{aligned}
$$

by (8). We thus see that the reproducing kernel of $L_{\mathrm{ph}}^{2}(\Omega, d \nu)$ must be given by

$$
H_{x}(y) \equiv H(y, x)=K(x, y)+K(y, x)-\frac{1}{\nu(\Omega)}
$$


Proof of Theorem 1, In view of (9), $\widetilde{T}_{f}=0$ if and only if

$$
\begin{aligned}
0 & =\left\langle f H_{x}, H_{x}\right\rangle=\int_{\Omega} f \cdot\left|H_{x}\right|^{2} d \nu=\int_{\Omega} f \cdot\left(K_{x}+\overline{K_{x}}-\frac{1}{\nu(\Omega)}\right)^{2} d \nu \\
& =\int_{\Omega} f \cdot\left(K_{x}^{2}+{\overline{K_{x}}}^{2}+\frac{1}{\nu(\Omega)^{2}}-2 \frac{1}{\nu(\Omega)} K_{x}-2 \frac{1}{\nu(\Omega)} \overline{K_{x}}+2\left|K_{x}\right|^{2}\right) d \nu
\end{aligned}
$$

for all $x$. Since the mapping $x \mapsto \overline{K_{x}}$ is holomorphic, this means that

$$
\int_{\Omega} f\left|K_{x}\right|^{2} d \nu=a(x)+\overline{b(x)} \quad \forall x \in \Omega
$$

where

$$
\begin{aligned}
& a(x)=\int_{\Omega} f \cdot\left({\overline{K_{x}}}^{2}-2 \frac{1}{\nu(\Omega)} \overline{K_{x}}+\frac{1}{2} \frac{1}{\nu(\Omega)^{2}}\right) d \nu, \\
& b(x)=\int_{\Omega} \bar{f} \cdot\left({\overline{K_{x}}}^{2}-2 \frac{1}{\nu(\Omega)} \overline{K_{x}}+\frac{1}{2} \frac{1}{\nu(\Omega)^{2}}\right) d \nu
\end{aligned}
$$

are holomorphic functions on $\Omega$. It is well known that if a function $F(x, y)$ is holomorphic in $x$ and $\bar{y}$ and vanishes for $x=y$, then it must vanish identically ([BM], Proposition II.4.7). Thus we even have

$$
\int_{\Omega} f K_{y} \overline{K_{x}} d \nu=a(x)+\overline{b(y)} \quad \forall x, y \in \Omega .
$$

Taking in particular $y=0, x=0$, and $x=y=0$, respectively, we get

$$
\begin{gathered}
\frac{1}{\nu(\Omega)} \int_{\Omega} f \overline{K_{x}} d \nu=a(x)+\overline{b(0)}, \\
\frac{1}{\nu(\Omega)} \int_{\Omega} f K_{y} d \nu=a(0)+\overline{b(y)}, \\
\frac{1}{\nu(\Omega)^{2}} \int_{\Omega} f d \nu=a(0)+\overline{b(0)},
\end{gathered}
$$

by (8). Consequently,

$$
\int_{\Omega} f \cdot\left(K_{y}-\frac{1}{\nu(\Omega)}\right)\left(\overline{K_{x}-\frac{1}{\nu(\Omega)}}\right) d \nu=0 \quad \forall x, y \in \Omega .
$$

Now, by (8) again, for any $f \in L_{\text {hol }}^{2}(\Omega, d \nu)$ we have $f-f(0)=P_{1^{\perp}} f$, the orthogonal projection of $f$ onto the orthogonal complement $1^{\perp}$ of the function 1 in $L_{\text {hol }}^{2}(\Omega, d \nu)$. Thus the last equality means that

$$
\left\langle P_{\mathbf{1}^{\perp}} T_{f} P_{\mathbf{1}^{\perp}} K_{y}, K_{x}\right\rangle=0 \quad \forall x, y \in \Omega .
$$

Since $T_{f}$ is bounded (in fact, $\left\|T_{f}\right\| \leq\|f\|_{\infty}$ by the definition of the Toeplitz operator) and the linear combinations of $K_{x}, x \in \Omega$, are dense in $L_{\text {hol }}^{2}(\Omega, d \nu)$, it follows that $\left\langle T_{f} P_{\mathbf{1}^{\perp}} f, P_{\mathbf{1}^{\perp}} g\right\rangle=0$ for all $f, g \in L_{\mathrm{hol}}^{2}$. In particular,

$$
\left\langle T_{f} z^{\alpha}, z^{\beta}\right\rangle=\int_{\Omega} f(z) z^{\alpha} \bar{z}^{\beta} d \nu(z)=0
$$

for any multiindices $\alpha, \beta$ with $|\alpha|,|\beta| \geq 1$. It follows that $\int_{\Omega} f(z)|z|^{2} p(z, \bar{z}) d \nu=0$ for any polynomial $p$ in $z, \bar{z}$, and, hence, by the Stone-Weierstrass theorem for any continuous function $p$ on $\Omega$. Thus $f(z)|z|^{2}=0$ a.e., hence $f=0$ a.e., and the proof is complete. 
Before continuing, we pause to mention a situation when the hypothesis of the boundedness of $\Omega$ in Theorem 1 can be dropped. Recall that a domain $\Omega \subset \mathbf{C}^{n}$ is called complete Reinhardt if $x \in \Omega$ and $\left|y_{j}\right| \leq\left|x_{j}\right| \forall j$ imply $y \in \Omega$. In particular, such domains are invariant under the rotations

$$
z \mapsto\left(z_{1} e^{i \theta_{1}}, z_{2} e^{i \theta_{2}}, \ldots, z_{n} e^{i \theta_{n}}\right) \quad \forall \theta_{1}, \ldots, \theta_{n} \in \mathbf{R} .
$$

Theorem 4. Let $\Omega \subset \mathbf{C}^{n}$ be a complete Reinhardt domain (not necessarily bounded) and $\nu$ a finite measure on $\Omega$ invariant under the rotations (13). Then on $L_{\mathrm{ph}}^{2}(\Omega, d \nu)$,

$$
\widetilde{T}_{f}=0 \Longrightarrow T_{f}=0 \text {. }
$$

Proof. As in the preceding proof, we arrive at the conclusions (11), (12), and $P_{1} \perp T_{f} P_{1} \perp=0$; however, in view of the possible unboundedness of $\Omega$, the monomials $z^{\alpha}$ need no longer be contained in $L_{\text {hol }}^{2}(\Omega, d \nu)$, and thus a different argument is required to conclude the proof. Differentiating (11) on both sides, we see that for any multiindices $\alpha, \beta$ with $|\alpha|,|\beta| \geq 1$,

$$
0=\frac{\partial^{|\alpha|+|\beta|}}{\partial x^{\alpha} \partial \bar{x}^{\beta}} \int f\left|K_{x}\right|^{2} d \nu=\int f \frac{\partial^{|\beta|} K_{x}}{\partial \bar{x}^{\beta}} \frac{\overline{\partial^{|\alpha|} K_{x}}}{\partial \bar{x}^{\alpha}} d \nu
$$

(The differentiation under the integral sign is legitimate since, in view of the holomorphic dependence of $\bar{K}_{x}$ on $x$, we may use Cauchy's formula to replace the differentiation by an integration over a small contour around $x$, and then apply Fubini's theorem.) However, by the radial symmetry of $\Omega$ and $d \nu$ the monomials are a basis of $L_{\text {hol }}^{2}$; thus

$$
K(y, x)=\sum_{\alpha} \frac{y^{\alpha} \bar{x}^{\alpha}}{\left\|z^{\alpha}\right\|^{2}}, \quad \text { whence }\left.\quad \frac{\partial^{|\alpha|} K(y, x)}{\partial \bar{x}^{\alpha}}\right|_{x=0}=\frac{\alpha !}{\left\|z^{\alpha}\right\|^{2}} \cdot y^{\alpha},
$$

with $1 /\left\|z^{\alpha}\right\|^{2}$ interpreted as zero if $z^{\alpha} \notin L^{2}(\Omega, d \nu)$. Setting $x=0$ in (14) we therefore obtain

$$
0=\int_{\Omega} f(y) y^{\beta} \bar{y}^{\alpha} d \nu(y)
$$

for all multiindices $\alpha, \beta$ such that $|\alpha|,|\beta| \geq 1$ and $z^{\alpha}, z^{\beta} \in L_{\text {hol }}^{2}(\Omega, d \nu)$. Next, substituting (12) into (10) we obtain

$$
\int_{\Omega} f \cdot\left(K_{x}^{2}+{\overline{K_{x}}}^{2}-\frac{1}{\nu(\Omega)^{2}}\right) d \nu=0 \quad \forall x .
$$

Differentiating again we see that for any multiindex $\alpha$ with $|\alpha| \geq 1$,

$$
\begin{aligned}
0 & =\left.\frac{\partial^{|\alpha|}}{\partial x^{\alpha}} \int_{\Omega} f \cdot{\overline{K_{x}}}^{2} d \nu\right|_{x=0} \\
& =\left.\sum_{\gamma \subset \alpha}\left(\begin{array}{l}
\alpha \\
\gamma
\end{array}\right) \int_{\Omega} f \cdot \frac{\partial^{|\gamma|}}{\partial x^{\gamma}} \overline{K_{x}} \cdot \frac{\partial^{|\alpha-\gamma|}}{\partial x^{\alpha-\gamma}} \overline{K_{x}}\right|_{x=0} d \nu \\
& =\sum_{\gamma \subset \alpha} \frac{\alpha !}{\left\|z^{\gamma}\right\|^{2}\left\|z^{\alpha-\gamma}\right\|^{2}} \int_{\Omega} f(y) \bar{y}^{\alpha} d \nu(y)
\end{aligned}
$$

The constant in front of the integral is positive whenever there exist $\beta$ and $\gamma$ such that $\beta+\gamma=\alpha$ and $z^{\beta}, z^{\gamma} \in L_{\text {hol }}^{2}(\Omega, d \nu)$; hence

$$
\int_{\Omega} f(y) \bar{y}^{\alpha} d \nu(y)=0
$$


for all $\alpha$ as above and such that $|\alpha| \geq 1$. Similarly for $\int_{\Omega} f(y) y^{\alpha} d \nu(y)$. Finally, taking $x=0$ in (10) yields $\int_{\Omega} f d \nu=0$, by (8). Thus we see that

$$
\int_{\Omega} f(y) y^{\alpha} \bar{y}^{\beta} d \nu(y)=\int_{\Omega} f(y) y^{\alpha} y^{\beta} d \nu(y)=0
$$

for all $\alpha, \beta$ such that $z^{\alpha}, z^{\beta} \in L_{\mathrm{hol}}^{2}(\Omega, d \nu)$. Since for complete circular domains and measures $L_{\mathrm{hol}}^{2}$ is spanned by the polynomials contained in it (see, e.g. E1]), by (77) the span of $\left\{z^{\alpha}, \bar{z}^{\alpha}: \alpha\right.$ a multiindex such that $\left.z^{\alpha} \in L_{\text {hol }}^{2}(\Omega, d \nu)\right\}$ must be all of $L_{\mathrm{ph}}^{2}(\Omega, d \nu)$. Thus

$$
\int_{\Omega} f \phi \bar{\psi} d \nu=0 \quad \forall \phi, \psi \in L_{\mathrm{ph}}^{2}(\Omega, d \nu),
$$

that is, $T_{f}=0$ on $L_{\mathrm{ph}}^{2}(\Omega, d \nu)$.

Remark. In general, if $\Omega$ is not bounded, $T_{f}=0$ need not imply that $f=0$, even if the measure $\nu$ is finite. For instance, there exist Reinhardt domains of finite volume for which $L_{\mathrm{hol}}^{2}$ (and, hence, $L_{\mathrm{ph}}^{2}$ ) reduces to the constant functions (see Wiegerinck (Wie]), and then $T_{f}=0$ whenever $\int_{\Omega} f=0$.

Remark. If $\nu(\Omega)=\infty$, then the formulas (8) and (9) still remain valid; only $\frac{1}{\nu(\Omega)}$ has to be interpreted as zero. However, the definition of the Berezin transform does not make sense at the origin since $K(0,0)=0$ by (8). Still, the argument used in the proof of Theorem 1 works without changes (just with $P_{1 \perp}$ interpreted as the identity operator, i.e. omitted), and thus the following assertion holds.

Proposition 5. Let $\Omega \subset \mathbf{C}^{n}$ be a complete circular domain (not necessarily bounded) and $\nu$ a measure on $\Omega$ (not necessarily finite) invariant under the rotations (13). Then on $L_{\mathrm{ph}}^{2}(\Omega, d \nu)$,

$$
\left\langle T_{f} H_{x}, H_{x}\right\rangle=0 \forall x \Longrightarrow T_{f}=0 .
$$

We now turn to the proof of Theorem 2, which in fact is a trivial application of the formula (9).

Proof of Theorem 2, By (19), (8) and (9),

$$
H_{h}(x, x) \approx 2 e^{\Phi(x) / h} h^{-n} \sum_{j=0}^{\infty} h^{j} b_{j}(x)-e^{\Phi(0) / h} h^{-n} \sum_{j=0}^{\infty} h^{j} b_{j}(0) .
$$

Observe that the origin is a strict global minimum of $\Phi$ : indeed, for any $x \neq 0$, the function of one complex variable $g_{x}(z):=\Phi(z x)$ is strictly subharmonic and depends only on $|z|$; thus by the sub-mean-value property of subharmonic functions $g_{x}(0)<g_{x}(1)$, or $\Phi(0)<\Phi(x)$. Consequently, if $x \neq 0$, then the second term in (15) is smaller by an exponential factor than the first, and so can be dropped.

Remark. In contrast to the holomorphic case, the coefficients of the asymptotic expansion (6) are not smooth but may have a jump discontinuity at the origin.

Before proceeding, we pause to recall some facts about the method of stationary phase. Let $f$ be a smooth function on $\mathbf{R}^{d}$ with compact support, and $S$ a smooth function such that

$$
S(y)=0, \quad \operatorname{Re} S(x)<0 \text { for } x \neq y, \quad \operatorname{det} S^{\prime \prime}(y) \neq 0 .
$$


Then as $h \searrow 0$,

$$
(\pi h)^{-d / 2} \int f(x) e^{S(x) / h} d x= \begin{cases}\sum_{j=0}^{\infty} h^{j} R_{j} f(y) & \text { if } S^{\prime}(y)=0 \\ O\left(h^{\infty}\right) & \text { if } S^{\prime}(y) \neq 0 .\end{cases}
$$

Here $R_{j}$ are some differential operators whose coefficients are given by expressions involving only $S$ and its derivatives. In particular,

$$
R_{0} f(y)=\operatorname{det} S^{\prime \prime}(y)^{-1 / 2} f(y) .
$$

See for instance Hörmander $\mathrm{Hrm}$, Section 7.7.

The assertion also remains in force for $f$ with not necessarily compact support, provided that the integral exists for some $h=h_{0}>0$ and "Re $S(x)$ is bounded away from 0 for $x$ away from $y$ " in the sense that $\operatorname{Re} S(x) \rightarrow 0$ implies $x \rightarrow y$.

\section{Proof of Theorem 3}

Recall that a bounded domain $\Omega \subset \mathbf{C}^{n}$ is called symmetric if for each $x \in \Omega$ there exists a holomorphic mapping $s_{x}: \Omega \rightarrow \Omega$ such that $s_{x} \circ s_{x}$ is the identity and $x$ is an isolated fixed-point of $s_{x}$. The simplest example of such domains are the unit balls of $m \times M$ complex matrices (viewed as operators from $\mathbf{C}^{M}$ into $\mathbf{C}^{m}$, with operator norm); in particular, for $M=1$ we recover the unit ball of $\mathbf{C}^{m}$, and $m=M=1$ gives the most basic bounded symmetric domain of all, the unit disc. It is further known that, using a suitable biholomorphic transformation if necessary, any bounded symmetric domain can be put into a canonical (so-called HarishChandra) realization which is circular (with respect to the origin) and convex in particular, which is complete Reinhardt. Throughout the rest of this section we will assume that $\Omega$ is in its Harish-Chandra realization.

Bounded symmetric domains have been completely classified by Cartan, and from our point of view here, their main virtue is that they resemble the unit disc very prominently as far as Bergman spaces are concerned, in the following sense. Let $K(x, y)$ be the (ordinary unweighted holomorphic) Bergman kernel of $\Omega$ (i.e. the reproducing kernel of $L_{\text {hol }}^{2}(\Omega)$ ). For each $\alpha \geq 1$, consider the measure

$$
d \mu_{\alpha}(z):=K(z, z)^{1-\alpha} d z .
$$

Then $\mu_{\alpha}(\Omega)<\infty$ and the reproducing kernel of $L_{\mathrm{hol}}^{2}\left(\Omega, d \mu_{\alpha}\right)$ is given by

$$
K_{\alpha}(x, y)=\frac{\mu_{1}(\Omega)^{\alpha}}{\mu_{\alpha}(\Omega)} K(x, y)^{\alpha} .
$$

Further, the measure $d \mu$ corresponding to the metric $g_{i \bar{j}}$ from the Introduction with $\Phi(z)=\log K(z, z)$ coincides (up to a constant factor) with $K(z, z) d z$. It follows that the associated Berezin transform (which we now denote by $B^{\text {hol }}$ to avoid confusion with the pluriharmonic Berezin transform to be discussed below) is given by

$$
B_{\alpha}^{\mathrm{hol}} f(y)=\frac{\mu_{1}(\Omega)^{\alpha}}{\mu_{\alpha}(\Omega)} \int_{\Omega} f(x)\left[\frac{|K(x, y)|^{2}}{K(x, x) K(y, y)}\right]^{\alpha} d \mu(x),
$$

and an application of the stationary phase method leads to the asymptotic expansion (2) from Theorem Q:

$$
B_{\alpha}^{\mathrm{hol}} f=\sum_{j=0}^{\infty} \alpha^{-j} Q_{j} f \quad \text { as } h=1 / \alpha \searrow 0 .
$$


For $\Omega=\mathbf{D}$, the unit disc, $K(x, y)=\pi^{-1}(1-x \bar{y})^{-2}$, and (18) reduces to the well-known fact that

$$
K_{\alpha}(x, y)=\frac{2 \alpha-1}{\pi}(1-x \bar{y})^{-2 \alpha} .
$$

For the unit balls of complex $m \times M$ matrices, $K(x, y)=$ const $\cdot \operatorname{det}\left(1-y^{*} x\right)^{-m-M}$. For a general bounded symmetric domain, it is known that $1 / K(x, y)$ is a polynomial in $x, \bar{y}$ such that $1 / K(x, x)$ attains its maximum $1 / K(0,0)=\operatorname{vol}(\Omega)$ only at the origin and vanishes at the boundary; further, at $z=0$

$$
K(z, z)=K(0,0)+\gamma|z|^{2}+O\left(|z|^{4}\right), \quad \gamma>0 .
$$

We refer to Ara for further information on bounded symmetric domains.

Another example of a situation where (10)-(2) can be made fairly explicit is $\Omega=\mathbf{C}^{n}$ and $\Phi(z)=|z|^{2}$. The corresponding spaces $L_{\mathrm{hol}, h}^{2}$ are then the Fock (or Segal-Bargmann) spaces

$$
L_{\text {hol }}^{2}\left(\mathbf{C}^{n}, e^{-|z|^{2} / h}\right)
$$

of entire functions square-integrable with respect to a Gaussian measure, with reproducing kernels

$$
K_{h}(x, y)=(\pi h)^{-n} e^{\langle x, y\rangle / h} .
$$

In this case the associated (holomorphic) Berezin transform is given by

$$
B_{h}^{\text {hol }} f(y)=\int_{\mathbf{C}^{n}} f(x) e^{-|x-y|^{2} / h} \frac{d x}{(\pi h)^{n}},
$$

which means that it is just the heat equation solution operator for time $t=h / 4$; thus

$$
B_{h}^{\text {hol }} f=e^{\Delta h / 4} f=\sum_{j=0}^{\infty} \frac{h^{j}}{j ! 4^{j}} \Delta^{j} f,
$$

which is the asymptotic expansion (2).

We now turn to the proof of Theorem 3 , concerning the corresponding pluriharmonic analogues

$$
\begin{aligned}
& L_{\mathrm{ph}}^{2}\left(\Omega, d \mu_{\alpha}\right) \quad(\Omega \text { a bounded symmetric domain, } \alpha=1 / h), \\
& L_{\mathrm{ph}}^{2}\left(\mathbf{C}^{n}, e^{-|z|^{2} / h}\right) \quad \text { (pluriharmonic Fock space), }
\end{aligned}
$$

of the last two spaces.

Proof of Theorem 3. Recall that, by definition,

$$
B_{h} f(y)=\frac{\left\langle f H_{y}, H_{y}\right\rangle}{H(y, y)},
$$

where, by (8) and (9),

$$
H_{y}(x) \equiv H_{h}(x, y)=K_{h}(x, y)+K_{h}(y, x)-K_{h}(0,0) .
$$

Let us first deal with the case of $\Omega=\mathbf{C}^{n}$. Then, as we have seen in the preceding paragraph,

$$
K_{h}(x, y)=(\pi h)^{-n} e^{\langle x, y\rangle / h} .
$$


If $y=0$, then $H_{h}(x, 0)=K_{h}(x, 0)$, so $B_{h} f(0)=B_{h}^{\text {hol }} f(0)$ coincides with the holomorphic Berezin transform. So let us assume that $y \neq 0$. Then $(\pi h)^{n} H(y, y)=$ $2 e^{|y|^{2} / h}-1=2 e^{|y|^{2} / h}\left[1+O\left(h^{\infty}\right)\right]$, so

$$
B_{h} f(y) \approx \frac{(\pi h)^{-n}}{2 e^{|y|^{2} / h}} \int_{\mathbf{C}^{n}} f(x)\left(e^{\langle x, y\rangle / h}+e^{\langle y, x\rangle / h}-1\right)^{2} e^{-|x|^{2} / h} d x .
$$

Expanding out the square, we obtain

$$
\begin{aligned}
B_{h} f(y) \approx \frac{(\pi h)^{-n}}{2 e^{|y|^{2} / h}} \int_{\mathbf{C}^{n}} f(x) & {\left[e^{2\langle x, y\rangle / h}+e^{2\langle y, x\rangle / h}+1-2 e^{\langle x, y\rangle / h}\right.} \\
& \left.-2 e^{\langle y, x\rangle / h}+2\left|e^{\langle x, y\rangle / h}\right|^{2}\right] e^{-|x|^{2} / h} d x .
\end{aligned}
$$

Let us now apply the stationary phase to each term. The contribution from the first one can be written as

$$
\frac{1}{2} e^{-|y|^{2} / h}(\pi h)^{-n} \int f(x) e^{2\langle x, y\rangle / h} e^{-|x|^{2} / h} d x .
$$

The phase function $S(x)=-|y|^{2}+2\langle x, y\rangle-|x|^{2}$ satisfies $S(y)=0, S^{\prime \prime}(y)=-I$, and $\operatorname{Re} S(x)=-|x-y|^{2}<0$ if $x \neq y$, but $S_{x}^{\prime}=2 \bar{y}-\bar{x}, S_{\bar{x}}^{\prime}=-x$, so $S^{\prime}(y) \neq 0$ (since $y \neq 0$ ). Thus by (16), the contribution (23) is $O\left(h^{\infty}\right)$. Similarly for the second term (where everything is just the complex conjugate). For the third term, we likewise have

$$
\frac{1}{2} e^{-|y|^{2} / h}(\pi h)^{-n} \int f(x) e^{-|x|^{2} / h} d x \approx \frac{1}{2} e^{-|y|^{2} / h} f(0)=O\left(h^{\infty}\right) .
$$

The contribution from the fourth term can be estimated as follows:

$$
\begin{aligned}
& \left|e^{-|y|^{2} / h}(\pi h)^{-n} \int f(x) e^{\langle x, y\rangle / h} e^{-|x|^{2} / h} d x\right| \\
& \quad \leq e^{-|y|^{2} / 2 h}(\pi h)^{-n} \int e^{-|x|^{2} / 2 h}|f(x)| e^{-|x-y|^{2} / 2 h} d x \\
& \quad \leq e^{-|y|^{2} / 2 h}(\pi h)^{-n} \int|f(x)| e^{-|x-y|^{2} / 2 h} d x .
\end{aligned}
$$

As the last integral is $\approx(2 \pi h)^{n}|f(y)|$ by the stationary phase method, this is again $\lesssim 2^{n} e^{-|y|^{2} / 2 h}|f(y)|=O\left(h^{\infty}\right)$. Similarly for the fifth term (where everything is just the complex conjugate of the fourth). Finally, the last term contributes

$$
e^{-|y|^{2} / h}(\pi h)^{-n} \int f(x)\left|e^{\langle x, y\rangle / h}\right|^{2} e^{-|x|^{2} / h} d x=B_{h}^{\mathrm{hol}} f(y) .
$$

Thus we see that, both for $y \neq 0$ and for $y=0$,

$$
B_{h} f(y)=B_{h}^{\text {hol }} f(y)+O\left(h^{\infty}\right),
$$

which establishes the claim for the Fock space in view of the asymptotic expansion (21) for $B^{\mathrm{hol}}$.

For bounded symmetric domains, the argument is completely parallel, only the role of the exponential $e^{\langle x, y\rangle}$ is taken over by the unweighted holomorphic Bergman kernel $K(x, y)$, the Lebesgue measure is replaced by $d \mu(z)=K(z, z) d z$, and the normalizing constants are different.

Namely, instead of (22) the reproducing kernels are now given by the formula (18) (with $\alpha=1 / h$ ). Introducing the notations

$$
k(x, y):=\mu_{1}(\Omega) K(x, y), \quad c_{\alpha}=1 / \mu_{\alpha}(\Omega),
$$


the latter can be written more compactly as

$$
K_{\alpha}(x, y)=c_{\alpha} k(x, y)^{\alpha} .
$$

Also, $1 / k(x, y)$ is a polynomial in $x$ and $\bar{y}$,

$$
k(z, z)>k(0,0)=1 \text { for } z \neq 0, \quad k(z, z) \rightarrow+\infty \text { as } z \rightarrow \partial \Omega,
$$

and (20) becomes

$$
k(x, x)=1+p|x|^{2}+O\left(|x|^{4}\right), \quad p>0 .
$$

If $y=0$, then again $H_{h}(x, 0)=K_{h}(x, 0)$ and $B_{h} f(0)=B_{h}^{\text {hol }} f(0)$ coincides with the holomorphic Berezin transform, so let $y \neq 0$. Then

$$
\frac{H_{h}(y, y)}{K_{h}(y, y)}=2-\frac{K_{h}(0,0)}{K_{h}(y, y)}=2-k(y, y)^{-\alpha} .
$$

Since $k(y, y)>1$ for $y \neq 0$, the second term is exponentially small and can thus be neglected. Consequently $(\alpha=1 / h)$,

$$
\begin{aligned}
B_{h} f(y) & =\frac{c_{\alpha}}{c_{1}^{\alpha}} \int_{\Omega} f(x) \frac{\left[k(x, y)^{\alpha}+k(y, x)^{\alpha}-1\right]^{2}}{2 k(y, y)^{\alpha}-1} k(x, x)^{-\alpha} d \mu(x) \\
& \approx \frac{c_{\alpha}}{c_{1}^{\alpha}} \int_{\Omega} f(x) \frac{\left[k(x, y)^{\alpha}+k(y, x)^{\alpha}-1\right]^{2}}{2 k(y, y)^{\alpha}} k(x, x)^{-\alpha} d \mu(x) .
\end{aligned}
$$

Expanding the square again yields

$$
\begin{aligned}
B_{h} f(y) \approx \frac{c_{\alpha}}{2 c_{1}^{\alpha} k(y, y)^{\alpha}} \int_{\Omega} & f(x)\left[k(x, y)^{2 \alpha}+k(y, x)^{2 \alpha}+1-2 k(x, y)^{\alpha}\right. \\
& \left.-2 k(y, x)^{\alpha}+2\left|k(x, y)^{\alpha}\right|^{2}\right] k(x, x)^{-\alpha} d \mu(x) .
\end{aligned}
$$

We again proceed by applying the stationary phase method to each term; however, prior to that we need to know the asymptotic behaviour of $c_{\alpha}$. To that end, observe that it is immediate from the definition of the Berezin transform that $B_{h} \mathbf{1}=\mathbf{1}$. Thus taking $f=1$ and $y=0$ in (26), we get

$$
1 \approx \frac{c_{\alpha}}{c_{1}^{\alpha}} \int_{\Omega} k(x, x)^{-\alpha} d \mu(x) .
$$

In view of (24) and (25), the stationary phase method applies to the last integral and gives

$$
\int_{\Omega} k(x, x)^{-\alpha} d \mu(x) \approx(\pi h)^{n} \sum_{j=0}^{\infty} \gamma_{j} h^{j}
$$

as $h=1 / \alpha \searrow 0$, with some real numbers $\gamma_{j}, \gamma_{0}=1$. Thus

$$
\frac{c_{\alpha}}{c_{1}^{\alpha}}=O\left(h^{-n}\right) .
$$

The contribution from the first term in (27) is therefore

$$
\begin{aligned}
& \frac{c_{\alpha}}{2 c_{1}^{\alpha}} k(y, y)^{-\alpha} \int_{\Omega} f(x) k(x, y)^{2 \alpha} k(x, x)^{-\alpha} d \mu(x) \\
& =O\left(h^{-n}\right) k(y, y)^{-\alpha} \int_{\Omega} f(x) k(x, y)^{2 \alpha} k(x, x)^{-\alpha} d \mu(x) .
\end{aligned}
$$

The phase function in the last integral is

$$
S(x)=\log \frac{k(x, y)^{2}}{k(x, x) k(y, y)}=\log \frac{K(x, y)^{2}}{K(x, x) K(y, y)} .
$$


By the Cauchy-Schwarz inequality, the real part of this is $\leq 0$, with equality taking place only if $K_{x}$ and $K_{y}$ are linearly dependent, i.e. if $a f(x)+b f(y)=0$ for some $a, b \in \mathbf{C}$, not both zero, for all $f \in L_{\text {hol }}^{2}(\Omega)$. Taking for $f$ the function 1 shows that $a+b=0$, and taking for $f$ the coordinate functions then shows that $x=y$. Thus $\operatorname{Re} S$ indeed attains a unique global maximum at the point $y$. Also, by (24), $\operatorname{Re} S(x)$ tends to $-\infty$ as $x$ tends to the boundary. Finally, it can be shown that $S^{\prime \prime}(y)$ is invertible. Thus the stationary phase method applies to the integral in (28); so let us see if $S$ has a critical point at $y$. Clearly,

$$
S_{\bar{x}}^{\prime}(y)=\frac{\partial_{\bar{y}} k(y, y)}{k(y, y)}
$$

Since $k(y, y)$ is real-valued, this can vanish only if $\nabla k(y, y)=0$. We claim that this can happen only for $y=0$. To see this, observe first of all that owing to the circularity of $\Omega$ we can choose an orthogonal basis $\phi_{0}, \phi_{1}, \ldots$ of $L_{\text {hol }}^{2}(\Omega)$ such that $\phi_{0}=1$ and $\phi_{j}, j \geq 1$, are homogeneous polynomials of degrees $>1$. Since

$$
K(x, x)=\sum_{j} \frac{\left|\phi_{j}(x)\right|^{2}}{\left\|\phi_{j}\right\|^{2}}
$$

it follows that for any $t>0$,

$$
K(t x, t x)=\sum_{j} t^{2 j} \frac{\left|\phi_{j}(x)\right|^{2}}{\left\|\phi_{j}\right\|^{2}}
$$

that is,

$$
k(t x, t x)=\|\mathbf{1}\|^{2} \sum_{j} t^{2 j} \frac{\left|\phi_{j}(x)\right|^{2}}{\left\|\phi_{j}\right\|^{2}} .
$$

If $\phi_{j}(x)=0 \forall j \geq 1$ for some $x$, then, since $\phi_{j}$ are a basis, $f(x)=f(0)$ for all $f \in L_{\text {hol }}^{2}(\Omega)$, which implies that $x=0$. (Just take for $f$ the coordinate functions.) Thus for $x \neq 0$, some $\phi_{j}(x), j \geq 1$, is always nonzero, which implies that (30) is a strictly increasing function of $t$, and, consequently, $\nabla k(x, x)$ cannot vanish.

Thus $y$ is not a critical point of $S$, and therefore the contribution from (28) is $O\left(h^{\infty}\right)$.

The second term is handled similarly (just taking complex conjugates). The third term in (27) contributes

$$
\frac{c_{\alpha}}{2 c_{1}^{\alpha}} k(y, y)^{-\alpha} \int_{\Omega} f(x) k(x, x)^{-\alpha} d \mu(x) \approx \frac{1}{2} k(y, y)^{-\alpha} f(0),
$$

which is again $O\left(h^{\infty}\right)$ as $k(y, y)>1$. The contribution from the fourth term can be estimated in a similar way as for the Fock space:

$$
\begin{aligned}
\mid \frac{c_{\alpha}}{c_{1}^{\alpha}} & k(y, y)^{-\alpha} \int_{\Omega} f(x) k(x, y)^{\alpha} k(x, x)^{-\alpha} d \mu(x) \mid \\
& \leq \frac{c_{\alpha}}{c_{1}^{\alpha}} k(y, y)^{-\alpha} \int_{\Omega}|f(x)|\left|k(x, y)^{\alpha}\right| k(x, x)^{-\alpha / 2} d \mu(x) \\
& =\frac{c_{\alpha}}{c_{1}^{\alpha}} k(y, y)^{-\alpha / 2} \int_{\Omega}|f(x)| e^{\alpha s(x) / 2} d \mu(x),
\end{aligned}
$$

where $s(x)$ is the real part of the phase function $S(x)$ in (29). As we have seen there, $s$ has a unique global maximum (which thus must be a critical point) at $y$ and decays to $-\infty$ at the boundary. Thus the last integral is susceptible to the 
stationary phase method, which shows that it is $O\left(h^{n}\right)|f(y)|$ as $h=1 / \alpha \searrow 0$. Owing to the extra factor of $k(y, y)^{-\alpha / 2}$, it therefore again follows that this contribution is also $O\left(h^{\infty}\right)$. Finally, the fifth term in (27) is handled similar to the fourth one (passing to complex conjugates), and the last term is again nothing but the holomorphic Berezin transform $B_{h}^{\text {hol }} f(y)$. Thus we again arrive at the fact that, both for $y \neq 0$ and for $y=0$,

$$
B_{h} f(y)=B_{h}^{\text {hol }} f(y)+O\left(h^{\infty}\right),
$$

and the proof is complete.

\section{Concluding Remarks}

In a way, our Theorems 13 raise more questions than they answer. First of all, it is not clear whether the results are anomalies whose validity stems from the abundant symmetries of the domains, or whether they hold in more general settings. For instance, does Theorem 1 hold for the Toeplitz operators on the pluriharmonic Bergman space on a general smoothly bounded strictly pseudoconvex domain in $\mathbf{C}^{n}$ ? Or does Theorem 3 hold for the pluriharmonic analogues of the spaces $L_{\text {hol }}^{2}\left(\Omega, e^{-\Phi / h} d \mu\right)$ from the traditional Berezin and Berezin-Toeplitz quantizations? Finally, for domains $\Omega \subset \mathbf{C}^{n}$ with $n \geq 2$ one can also consider instead of $L_{\mathrm{ph}}^{2}$ the analogous spaces $L_{\text {harm }}^{2}$ of harmonic functions; in this case, it even makes sense to study the problem not only for pseudoconvex domains in $\mathbf{C}^{n}$, which are the natural arena for holomorphic functions, but for any open set $\Omega \subset \mathbf{R}^{n}$. In that setting, one can obtain an analogue of Theorem 3 for the "harmonic Fock spaces"

$$
L_{\text {harm }}^{2}\left(\mathbf{R}^{n}, e^{-|z|^{2} / h} d z\right)
$$

on $\mathbf{R}^{n}$ (see [E4]); however, currently it is not even known whether an analogue of Theorem 3 holds for the unit ball of $\mathbf{R}^{n}$.

In the holomorphic case, we have outlined in the beginning of Section 3 the proofs of our Theorem Q from the Introduction for the case of $\Omega=\mathbf{C}^{n}$ and of $\Omega$ a bounded symmetric domain. In the remaining case of a smoothly bounded strictly pseudoconvex domain, the asymptotics of the weighted Bergman kernels, of the Berezin transform and of the Toeplitz operators can be derived from the boundary behaviour of the Szegö kernel of the "inflated" domain $\widetilde{\Omega}=\left\{(x, t) \in \Omega \times \mathbf{C}:|t|^{2}<\right.$ $\left.e^{-\Phi(x)}\right\}$, using the formula of Forelli-Rudin-Ligocka and the Fefferman-Boutet de Monvel-Sjöstrand theorem. Namely, the hypotheses ensure that $\widetilde{\Omega}$ is smoothly bounded and strictly pseudoconvex and admits $r(x, t):=e^{-\Phi(x)}-|t|^{2}$ as a defining function. Its boundary $\mathcal{X}=\partial \widetilde{\Omega}$ is a smooth compact manifold, and $\alpha=\operatorname{Im} \partial r$ is a contact form on $\mathcal{X}$ (i.e. $\alpha \wedge(d \alpha)^{n-1}$ is a nonvanishing volume element). Let $H^{2}(\mathcal{X})$ be the Hardy subspace of all functions in $L^{2}(\mathcal{X})$ that extend holomorphically into $\widetilde{\Omega}$. According to a formula of Forelli, Rudin and Ligocka, the reproducing kernel $K_{\mathcal{X}}$ of $H^{2}(\mathcal{X})$ - the Szegö kernel — satisfies

$$
K_{\mathcal{X}}((x, t),(y, s))=\frac{1}{2 \pi n !} \sum_{k=0}^{\infty}(t \bar{s})^{k} K_{1 /(k+n+1)}(x, y) .
$$

On the other hand, by results of Fefferman, Boutet de Monvel and Sjöstrand,

$$
\left.K_{\mathcal{X}}\right|_{\text {diagonal }}=\frac{a}{r^{n+1}}+b \log r, \quad \text { where } a, b \in C^{\infty}(\overline{\widetilde{\Omega}}) .
$$


Employing the usual Cauchy estimates for the function $f_{x}(t \bar{s}):=K_{\mathcal{X}}((x, t),(x, s))$ of one complex variable on the disc $|t \bar{s}|<e^{-\Phi(x)}$, the expansion (11) is obtained (where $h=1 /(k+n+1), k \rightarrow \infty$ ). In fact, this even gives a similar expansion for $K_{h}(x, y)$ for $(x, y) \in \Omega \times \Omega$ close to the diagonal, and (2) then follows by an application of the stationary phase method. Finally, (3) can be proved using the Boutet de Monvel-Guillemin theory of generalized Toeplitz operators (with pseudodifferential symbols). See, e.g. [E2 for the details. (A completely analogous result also holds if $\Omega$ is an arbitrary Kähler manifold such that the second cohomology class $[\omega]$ of the Kähler form $\omega$ is integral, only one has to use sections of line bundles instead of functions; see BMS, Zel].)

It should be noted that the Forelli-Rudin-Ligocka formula also holds for the pluriharmonic Bergman spaces: if we denote by $H_{\mathrm{ph}}^{2}(\mathcal{X}), \mathcal{X}=\partial \widetilde{\Omega}$, the subspace in $L^{2}(\mathcal{X})$ of all functions that have a pluriharmonic extension inside $\widetilde{\Omega}$, then the reproducing kernel of $H_{\mathrm{ph}}^{2}(\mathcal{X})$ is given by

$$
K_{\mathcal{X}}^{\mathrm{ph}}((x, t),(y, s))=\frac{1}{2 \pi n !} \sum_{j=-\infty}^{\infty}(s \bar{t})^{[j]} K_{1 /(|j|+n+1)}^{\mathrm{ph}}(x, y),
$$

where $z^{[j]}=z^{j}$ or $\bar{z}^{-j}$ according to whether $j \geq 0$ or $<0$, and $K_{1 / m}^{\mathrm{ph}}(x, y)$ is the reproducing kernel of $L_{\mathrm{ph}}^{2}\left(\Omega, e^{-m \Phi} d \mu\right)$. Thus in principle we can again get the asymptotics as $m \rightarrow+\infty$ of $K_{1 / m}^{\mathrm{ph}}$, and of the pluriharmonic Berezin transform $B_{1 / m}^{\mathrm{ph}}$, from the boundary singularity of $K_{\mathcal{X}}^{\mathrm{ph}}$. Unfortunately, what is missing is the pluriharmonic analogue of Fefferman's theorem, i.e. the description of the boundary singularity of the pluriharmonic Szegö or Bergman kernels.

Similarly, it seems unknown what is the boundary singularity of the harmonic Bergman (or Szegö) kernel of a domain in $\mathbf{R}^{n}$. (There exist optimal estimates for the boundary growth, though; see $\mathrm{KK}$.) However, in this case there is no analogue of the Forelli-Rudin-Ligocka formula.

\section{ACKNOWLEDGEMENT}

Some of the above results were presented in the fall of 2005 at the conference on Complex Analysis from the Geometric Viewpoint in Leipzig and at the Hayama Symposium on Several Complex Variables. The author thanks the organizers for the invitations.

\section{REFERENCES}

[Ara] J. Arazy: A survey of invariant Hilbert spaces of analytic functions on bounded symmetric domains, Multivariable operator theory (R.E. Curto, R.G. Douglas, J.D. Pincus, N. Salinas, editors), Contemp. Math. 185, Amer. Math. Soc., Providence, 1995, pp. 7-65. MR 1332053 (96e:46034)

[Ber] F.A. Berezin: Quantization in complex symmetric spaces, Math. USSR Izvestiya 9 (1975), 341-379. MR0508179 (58:22691)

[BM] S. Bochner, W.T. Martin, Several complex variables, Princeton University Press, Princeton, NJ, 1948. MR0027863(10:366a)

[BMS] M. Bordemann, E. Meinrenken, M. Schlichenmaier: Toeplitz quantization of Kähler manifolds and $g l(n), n \rightarrow \infty$ limits, Comm. Math. Phys. 165 (1994), 281-296. MR1301849 (96f:58067)

[BLU] D. Borthwick, A. Lesniewski, H. Upmeier: Non-perturbative deformation quantization on Cartan domains, J. Funct. Anal. 113 (1993), 153-176. MR1214901(94d:47065) 
[Cob] L.A. Coburn: Deformation estimates for the Berezin-Toeplitz quantization, Comm. Math. Phys. 149 (1992), 415-424. MR1186036 (93j:47038)

[E1] M. Engliš: Berezin quantization and reproducing kernels on complex domains, Trans. Amer. Math. Soc. 348 (1996), 411-479. MR1340173 (96j:32008)

[E2] M. Engliš: Weighted Bergman kernels and quantization, Comm. Math. Phys. 227 (2002), 211-241. MR1903645 (2003f:32003)

[E3] M. Engliš: Berezin and Berezin-Toeplitz quantizations for general function spaces, Rev. Mat. Complut. 19 (2006), 385-430. MR2241437 (2007d:53152)

[E4] M. Engliš: Berezin transform on the harmonic Fock space, in preparation.

[Hrm] L. Hörmander, The analysis of linear partial differential operators, vol. I, Grundlehren der mathematischen Wissenschaften 256, Springer-Verlag, Berlin - Heidelberg - New York - Tokyo, 1985.

[KK] H. Kang, H. Koo: Estimates of the harmonic Bergman kernel on smooth domains, J. Funct. Anal. 185 (2001), 220-239. MR1853757 (2002h:46042)

[Wie] J.J.O.O. Wiegerinck: Domains with finite dimensional Bergman space, Math. Z. 187 (1984), 559-562. MR760055 (86a:32049)

[Zel] S. Zelditch: Szegö kernels and a theorem of Tian, Int. Math. Res. Not. 6 (1998), 317-331. MR.1616718 (99g:32055)

Mathematical Institute, Czech Academy of Sciences, Žitná 25, 11567 Prague 1, Czech Republic

E-mail address: englis@math.cas.cz 